
du F.L.E. (18e et 19e siècles)

\title{
La littérature au service de la langue: Les Exercices français de Pierre-Joseph Baudet destinés à la jeunesse néerlandaise (1834-1844)
}

\section{Marie-Christine Kok Escalle}

\section{CpenEdition}

Édition électronique

URL : https://journals.openedition.org/dhfles/3019

DOI : $10.4000 /$ dhfles.3019

ISSN : 2221-4038

\section{Éditeur}

Société Internationale pour l'Histoire du Français Langue Étrangère ou Seconde

Édition imprimée

Date de publication : 1 décembre 1999

Pagination : p.161-173

ISSN : 0992-7654

Référence électronique

Marie-Christine Kok Escalle, « La littérature au service de la langue: Les Exercices français de PierreJoseph Baudet destinés à la jeunesse néerlandaise (1834-1844) », Documents pour I'histoire du français langue étrangère ou seconde [En ligne], 24 | 1999, mis en ligne le 22 janvier 2015, consulté le 27 mai 2021. URL : http://journals.openedition.org/dhfles/3019; DOI : https://doi.org/10.4000/dhfles.3019

Ce document a été généré automatiquement le 27 mai 2021.

(c) SIHFLES 


\title{
La littérature au service de la langue: Les Exercices français de Pierre-Joseph Baudet destinés à la jeunesse néerlandaise (1834-1844)
}

\author{
Marie-Christine Kok Escalle
}

1 Pierre-Joseph Baudet, né à Braine-le-Comte dans le Hainaut belge en 1778, est mort à Utrecht, aux Pays-Bas, en 1858 à l'âge de 80 ans. On le trouve à Deventer en 1798. Il sera instituteur et maître de français à Leeuwarden, à Vaassen près de Deventer, et à 's Graveland; puis, à Utrecht, il enseignera les mathématiques à la fin de sa vie, sans grand succès si l'on considère les résultats de ses élèves aux examens.

2 Auteur de manuels de mathématiques, d'algèbre, d'arithmétique, d'astronomie, il offre aussi pour l'apprentissage de la langue française divers écrits didactiques destinés aux élèves hollandais -petits ou grands, débutants ou avancés. Sa pratique didactique pour l'enseignement des sciences paraît nouvelle aux historiens de l'enseignement des mathématiques. Sa méthode qui est une méthode appliquée est originale aussi pour l'enseignement des langues étrangères; sous le nom d'Exercices il offre une méthode totale d'apprentissage de la langue française pour les Hollandais (grammaire, vocabulaire, culture).

3 La langue française y est présentée à la fois comme un parler et comme un «se conduire», une forme d'éloquence et un contenu moral et social. L'un et l'autre s'appuient sur une référence littéraire, «les bons auteurs» ou «les bons écrivains», tout comme le faire de Baudet lui-même pour lequel il renvoie à Boileau et à Montaigne. Ainsi, dans ses Principes abrégés de la langue française ou Elémens de grammaire à l'usage des jeunes gens hollandais (Deventer J. H. de Lange, 1810, 8), P. J. Baudet souligne l'imbrication étroite de la langue et la littérature, donnant au style une place importante dans l'apprentissage d'une langue étrangère; la citation de Boileau ornant la page de titre «Sans la langue, en un mot, l'auteur le plus divin est toujours, quoi qu'il fasse, un méchant écrivain» en assure le message. Dans la préface, il livre aussi une clé 
de lecture de sa pratique didactique: «Pour donner plus d'autorité aux règles je les ai appuyées de phrases puisées dans les auteurs les plus accrédités pour la beauté du stile et la pureté de la morale» (iii-iv). L'apprentissage de la langue française concerne donc bien la forme et le fond. "Si j'ai su présenter aux jeunes gens une méthode facile d'apprendre les premiers élémens de la Langue Française; si j'ai pu fortifier en eux l'amour du bon et de l'honnête en leur offrant des exemples d'une morale solide, j'aurai atteint mon but et j'aurai lieu d'espérer les suffrages du public» (iv). C'est cette imbrication que nous nous proposons d'étudier.

Après avoir esquissé les principes pédagogiques de Baudet et la place que la littérature occupe dans sa méthode au service de l'apprentissage de la langue, nous analyserons la fonction que Baudet attribue à la littérature. D'une part, la perfection classique est d'un point de vue linguistique la forme idéale, d'autre part, elle véhicule les valeurs culturelles modèles et incarne le comportement moral social idéal.

5 Le pédagogue Baudet, sur lequel nous n'avons pas encore trouvé d'études, alors qu'il a publié beaucoup de manuels pour l'apprentissage des mathématiques, de l'astronomie, etc, a laissé nombre de manuels pour l'apprentissage de la langue française. Après les Principes abrégés... (ouvr. cité) publiés en 1810, paraît dix ans plus tard, sans doute pour les besoins de ses élèves, un Petit Recueil de Poésies à l'usage de l'enfance (1820, Deventer, J. $\mathrm{H}$. De Lange, $\mathrm{I}^{\mathrm{e}}$ partie); la littérature offerte aux petits Hollandais comprend quelque huit fables de La Fontaine dont trois ${ }^{1}$ font partie du patrimoine scolaire des petits Français du $20^{e}$ siècle, suivies de trois courts textes de Racine (respectivement 6, 13 et 4 vers), de deux textes de Boileau et un autre de La Fontaine; le tout est couronné par un texte de Voltaire sur la mort de Coligny (20-22), ce qui renvoie à une mémoire historique commune aux Pays-Bas et à la France. Ces textes sont à connotation morale et incarnent les références classiques par excellence ${ }^{2}$. Dès le plus jeune âge donc, l'apprentissage de la langue française qui est l'objet de cet ouvrage comme en témoigne le lexique français-néerlandais que l'on trouve à la fin, est donc lié à des contenus moraux et à des noms d'écrivains classiques.

Fruit de la longue expérience de Baudet, maitre de langue française, huit volumes des Exercices français avec les indications grammaticales nécessaires et des explications au service de la jeunesse néerlandaise paraissent de 1834 (ou 1833) à 1844³. Ces Exercices ont été complétés, un an plus tard, par l'Alphabet français à l'usage des commençants ${ }^{4}$ puis une Suite à l'Alphabet dont l'objet est «d'exercer et d'enrichir la mémoire des élèves, en même temps qu'ils s'occupent de la partie grammaticale». Enfin, pour un public plus jeune, Baudet a écrit un Supplément à l'alphabet français à l'usage des commençants contenant une série d'exercices pour le plus jeune âge ${ }^{6}$.

7 Les livres d'Exercices ${ }^{7}$ de Baudet que nous avons choisi d'étudier ne sont ni une grammaire pure et simple, ni un livre de dialogues ou un manuel de conversation comme il y en a tant aux $17^{\mathrm{e}}$ et $18^{\mathrm{e}}$ siècles, qui exposent les principes théoriques et guident à la pratique de la langue. Ils ne sont pas non plus une anthologie de textes littéraires comme il commence à en exister en Europe au début du $19^{\mathrm{e}}$ siècle pour l'apprentissage du français langue étrangère ${ }^{8}$. Ils ne sont pas non plus un manuel élémentaire comme celui de Basedow et Wolke ${ }^{9}$ qui réunit les connaissances fondamentales que les élèves doivent apprendre, toutes les matières y étant abordées dans des leçons. Ils sont en ce sens une publication pédagogique originale puisqu'ils abordent tous les aspects que l'on trouve dans les différents manuels et offrent d'une certaine façon aux. apprenants de français une initiation à la vie. Ces Exercices sont non 
«un cours complet de Syntaxe,... [mais] un cours d'étude, et non un ouvrage scientifique qui offrît un répertoire de l'état de la science» (IV: 111). L'élève se trouve guidé dans son apprentissage de la langue française.

8 Baudet identifie sa pratique à celle des classiques. Mettre l'élève à l'étude, lui transmettre un savoir utile et lui faire acquérir un savoir-faire, lui offrir un passetemps agréable, voilà la prétention de Baudet qui se réfère à Boileau ou Montaigne pour justifier son faire. Il se compare à un charpentier dont le travail de construction doit tenir compte de l'ensemble dans lequel il construit. Le contexte d'apprentissage est donc à prendre en considération et en cela il se réfère à Montaigne pour qui: «Ce que nous avons à cette heure proposé, nous le changeons tantost, et tantost encores retournons sur nos pas: ce n'est que bransle et inconstance. Nous n'allons pas: on nous emporte» (IV: v).

Et Baudet précise: «... au $19^{\mathrm{e}}$ siècle, il ne faut pas enseigner une langue vivante, comme on enseignait le latin et le grec au $16^{\mathrm{e}}$, en faisant apprendre toutes les règles et toutes les exceptions relatives au substantif avant d'oser toucher à l'adjectif» (IV: vi).

Car il s'agit pour lui, d'offrir à l'élève un savoir et un savoir-faire, utile et agréable: « ... introduire la jeunesse studieuse dans la connaissance générale du Français en lui offrant une série d'occupations propres à exercer en même temps son jugement et sa mémoire, ... répandre des notions utiles ... offrir aux élèves une occupation agréable " (IV: vi). ... « La plupart des thèmes et des versions contiennent des choses instructives. ... Cette méthode réunit le double avantage de faire retenir en même temps les choses et les expressions, et de fournir un aliment à l'esprit. ... II importe que les jeunes gens trouvent dans leur travail un attrait qui occupe leur esprit lorsqu'ils sont seuls » (avant-propos VI: 1836).

11 Baudet fait preuve d'un certain optimisme en pensant «faire voir aux jeunes gens qu'il est une étude des mots et des expressions qui pourra encore les occuper agréablement après qu'ils auront quitté l'école» (avant-propos VII: 3). Il a choisi «dans une multitude de choses ... celles qui [lui] ont paru indispensables à la jeunesse hollandaise» [et souhaite qu'elles soient d'] 'une utilité proportionnée aux vœux qu'il fait pour «son bien-être» (avant-propos VII: 4).

12 Sa méthodologie est comparable à ce que $\mathrm{H}$. Christ ${ }^{10}$ appelle la méthode «synthétique». Après avoir énoncé une règle, Baudet donne un exemple puis un exercice qui permet à l'élève de découvrir lui-même les mécanismes de fonctionnement de la règle, de les comprendre et de réfléchir sur le système. Il combine íes compétences que les exercices sont censés faire acquérir, en complétant un exercice de traduction par un exercice d'application grammaticale par exemple. Sa pratique est certainement celle d'un précurseur; il s'inscrit en partie dans la ligne de la réforme qui, à la fin du $19^{\mathrm{e}}$ siècle, valorise la méthode directe qui «se caractérise par le refus de la traduction, ... [et] vise un apprentissage qui relie directement les expressions de la langue étrangère aux idées correspondantes sans que les signes de la langue maternelle s'intercalent $»^{11}$.

13 Baudet fait bien une large part à la traduction dans ses Exercices, mais sa réflexion sur la traduction et sur la pratique d'apprentissage sont tout-à-fait intéressantes. La langue est pour lui un outil conceptuel et culturel tout à la fois, un outil vivant qui évolue avec l'usage qu'on en fait. L'apprenant est donc confronté à la relation qui existe entre la norme et l'usage, et entre le fond et la forme. Ainsi commence-t-il la première partie du volume VII qui comprend «des exercices sur les synonymes et en général sur l'usage de la langue française»: « Pour bien s'exprimer dans une langue, il ne suffit pas d'observer 
rigoureusement les règles de la grammaire, il faut encore que chaque terme rende fidèlement la pensée, et que chaque expression soit autorisée par le bon usage. On ne parvient à ce degré de perfection que par la fréquentation des personnes qui possèdent le talent de bien s'exprimer, et par la lecture des bons écrivains ${ }^{12}$. Ici l'imitation l'emporte sur toutes les règles. Toutefois après avoir franchi les premières difficultés, c'est-à-dire après avoir parcouru la grammaire, il est important de s'acheminer vers le but que l'on se propose d'atteindre. Chaque langue a ses misères et ses ressources; chaque peuple a sa manière de voir et de s'exprimer. Il est donc souvent impossible de traduire une phrase mot-à-mot, soit qu'un mot d'une langue n'ait point d'équivalent dans une autre, soit qu'un peuple repousse comme inusitées des expressions approuvées par ses voisins » (Introduction à la première partie, VII: 5).

L'identification de Baudet, pédagogue et maître de langue française avec le poète ou écrivain classique se retrouve à plusieurs niveaux. D'une part, ses livres d'Exercices sont un produit comparable à un produit du classicisme, conforme à la poétique classique quant à l'objectif d'utilité et d'agrément; d'autre part, les matériaux utilisés pour l'apprentissage de la langue française, que sont les textes littéraires sont une référence constante au classicisme, dans son approche de l'art et de la nature.

Il fait donc œuvre d'art, comparable à la poétique classique qui «n'est utile qu'autant qu'elle est agréable» (Rapin, Réflexions, dans Kibedi-Varga, 1990; 156) ${ }^{13}$ et selon laquelle «toutes les connaissances qui peuvent éclairer notre âme sont à parler absolument, ou utiles, ou agréables» (La Mesnardière. La Poétique, 1640. préface, dans Kibedi-Varga, 1990: 51). Baudet enracine sa pratique dans la tradition classique, comparant son faire à celui de Boileau: "Dans le cours de cet ouvrage, j'ai tâché de répandre des notions utiles, et de fournir à la jeunesse une occupation agréable. Je n'hésite pas à m'appliquer ce vers de Boileau: c'est par là que je vaux, si je vaux quelque chose "(VI, avant-propos: iv).

Le faire du maitre est délibérément un faire à imiter. La littérature est mise au service de l'apprentissage de la langue, présentée comme un modèle à imiter, modèle de forme linguistique idéale, celle de la perfection classique.

Dans les livres d'Exercices, qui ne sont pas des livres de littérature, la littérature sert de guide à l'apprentissage de la forme linguistique; auteurs et textes littéraires sont des repères dans un champ d'étude du vocabulaire et du système grammatical. Les choix de textes et la sélection des auteurs classiques révèlent des normes de goût et d'idées propres à l'image de la langue française aux Pays-Bas dans la première moitié du $19^{\mathrm{e}}$ siècle. Le classicisme est le modèle à imiter.

Sous quelle forme apparaît la littérature? On trouve des textes d'auteurs littéraires donnés comme textes de versions à traduire en néerlandais, comme textes offerts à l'analyse grammaticale, et comme textes illustrant des structures syntaxiques ou des emplois sémantiques; au fil des exemples de grammaire ou d'usage, des auteurs sont cités comme modèles de performance d'écriture ou de comportement.

Les exemples littéraires, qui apparaissent dès le volume II, sont donnés comme objets de traduction -les versions- et d'analyse grammaticale -quatre phrases, citations d'auteurs, suivent chaque série de versions. Du volume II au volume VI on passe de quelques vers de Boileau, Voltaire et Racine, donnés à traduire (II: 98-100), à de longs textes littéraires. Les textes de version disparaissent dans les volumes VII et VIII, car dit Baudet, «l'étendue donnée aux thèmes et la conviction que les élèves expliquent un auteur m'ont engagé à supprimer les versions» (avant-propos VII: dd. 3 avril 1842). 

pour la traduction et l'analyse grammaticale, ils le sont aussi ${ }^{14}$ comme sujet d'illustration des règles et des usages grammaticaux; les principes et les pratiques de la langue sont présentés d'après des modèles littéraires. Le texte littéraire sert alors de support de message pour un idéal de forme (la structure grammaticale) et de fond (le contenu culturel) tout à la fois, comme en témoigne la phrase suivante qui illustre la règle selon laquelle les verbes impersonnels commandent le subjonctif (IV: 12): «Il importe que vous appreniez à rapporter les choses humaines aux ordres de la sagesse éternelle, dont elles dépendent» (Bossuet). définie par les théoriciens du classicisme, pour qui il s'agit d'imiter les Anciens, à savoir: «dans le sens le plus étendu, former son esprit, son langage, ses habitudes de concevoir, d'imaginer, de composer sur un modèle avec lequel on se sent quelque analogie» (Marmontel, Oeuvres, IX: 132, dans Kibedi-Varga 1990: 26). En prenant des exemples chez les écrivains classiques, Baudet invite l'élève à les imiter, à suivre leur pratique de la rhétorique qui «enseigne à arranger les discours et les arguments en vue de la persuasion, de la communication efficace» (Kibedi-Varga, 1990: 15).

Baudet justifie la syntaxe et la sémantique par l'usage littéraire de façon tant explicite qu'implicite: parler la langue française c'est faire comme [...] qui a écrit [...]. Les citations des auteurs qui forment le canon classique: Boileau, Racine, Voltaire, La Fontaine illustrent le principe (utilisation du signe pour la chose signifiée ou du nom abstrait pour le concret) et l'usage des expressions figurées -métaphore, allégorie, métonymie, synecdoque, ironie, hyperboles- ${ }^{15}$ Pour imiter de façon adéquate, il faut pouvoir comprendre; aussi Baudet livre des clés pour l'interprétation: «quand La Fontaine dit...., la pensée est que ...» (VIII: 12).

Imiter les classiques, c'est tenter de satisfaire aux exigences de perfection formelle, c'est suivre les principes de convenance. Baudet fait référence à Boileau pour justifier le soin mis à éviter les vices du discours; le vers de Boileau: «Fuyez des mauvais sons le concours odieux» sert à Baudet d'exemple pour expliquer le principe de l'élégance mis à l'honneur par les classiques: "La cacophonie est un vice qui consiste en un son désagréable, produit par la rencontre de lettres, de syllabes ou de mots que l'oreille repousse. Le vers cité en tête de cet article est une leçon que Boileau adresse aux poètes, et en effet, les bons écrivains évitent autant que possible la rencontre de syllabes semblables, comme: il commença sa harangue;... Toutefois il y aurait de l'injustice à vouloir astreindre des commençants à des règles aussi rigoureuses ... Voilà donc quelques préceptes. (VII: 179, «Appendice de quelques vices du discours»).

Les textes littéraires classiques cités dans les Exercices sont destinés à être imités dans leur forme et dans le message qu'ils transmettent. Les auteurs et leurs écrits sont cités pour cautionner une norme, celle de la beauté à laquelle est censée correspondre l'utilisation de la langue française qui consiste en une harmonie entre la forme et le fond. L'apprenant est appelé à répondre à cet idéal incarné par le classicisme, non plus de façon passive en traduisant des textes littéraires français dans sa langue maternelle, mais bien de façon active en devant traduire un texte néerlandais en français, en l'occurrence le thème (premier du volume VIII dont nous avons vu qu'il ne contient plus de version) dont la traduction est la suivante: «C'est à juste titre que Boileau dit: "Avant donc que d'écrire, apprenez à penser" (Art poétique). Il est vrai que Boileau adresse ce conseil aux poètes; mais ce conseil convient à tous ceux qui prennent la 
plume pour mettre leurs pensées sur papier même s'il s'agit d'écrire une lettre à un ami » (VIII: 33) [trad. MCKE]. Autrement dit, Baudet inscrit implicitement le travail de l'élève que représente l'apprentissage pratique de la langue française dans un cadre tracé par les auteurs classiques, et dont Boileau est la référence principale: lorsqu'on pratique la langue française on s'inscrit dans la tradition de Boileau et la ligne de l'Art Poétique.

Pour ce qui est de l'usage des expressions figurées, l'auteur classique semble bien être la norme. Boileau. Molière et Malherbe sont les modèles de l'harmonie nécessaire entre la forme («les belles phrases») et le fond («la justesse de la pensée»): «L'emploi des expressions figurées doit être dicté par le goût et par la connaissance de l'usage établi chez une nation. ... De plus il faut que cette expression soit autorisée par les bons écrivains» (VIII: 198). Ceux-ci sont, d'après la liste des proverbes cités, ceux du canon classique, à savoir Boileau, La Fontaine, Voltaire, Racine, auxquels s'ajoutent Molière et Corneille. Les expressions emblématiques citées qui manifestent un accord entre le bon sens et la vertu, font partie de la mémoire scolaire des petits Français du $20^{*}$ siècle, comme «Aux âmes bien nées», "La valeur n'attend pas le nombre des années» (Corneille) ou «Rien ne sert de courir il faut partir à point» (La Fontaine) (VIII: 199-200). Ici, l'apprenant est en fin de parcours de son apprentissage (volume VIII des Exercices) et Baudet peut espérer un résultat fructueux dans une production imitative des classiques. Le goût du beau, l'harmonie entre le fond et la forme se trouvent déjà dans le choix des poésies retenues pour les enfants, textes que l'on pourrait prendre comme modèle de classicisme. En effet, la simplicité, la clarté et l'élégance de l'écriture sont les caractéristiques de ces textes choisis chez La Fontaine, Boileau et Racine et dont plusieurs ont pour sujet le bonheur. La beauté Esthétique, celle de la langue écrite s'allie à la beauté dans le sens de la morale sociale: «Dans le monde il n'est rien de beau que l'équité» (Boileau) et «Ni l'or, ni la grandeur ne nous rendent heureux. ... Le sage y vit en paix, et méprise le reste» (La Fontaine) Petit Recueil de poésies à l'usage de l'enfance (1820: 17-18).

27 Tout cela est bien conforme au principe selon lequel les théoriciens du classicisme affirment que l'art doit «acheminer à la vertu» (Chapelain, cité par Kibedi-Varga, 1990: 31); le but de l'art est moral, il doit plaire et instruire. La poésie a donc un but utilitaire, celui d'instruire. Dans la tradition d'Aristote, elle doit enseigner le Bien, par l'intrigue, le caractère des personnages et le dénouement. «L'art précède et suscite la morale» (Kibedi-Varga, 1990: 32).

Avec Baudet, la littérature offerte à l'apprenant est présentée comme un modèle linguistique à imiter, la perfection formelle classique. Elle est aussi présentée comme un modèle socio-culturel à imiter; les textes choisis expriment en effet un comportement moral et social idéal, disent une norme destinée à devenir la norme pour l'élève, destinataire de l'ouvrage et incité à s'identifier au destinataire du texte littéraire. Elle est enfin utilisée comme support d'un certain bagage scientifique. Dans l'ensemble, le choix de textes littéraires dessine une certaine culture à s'approprier. Auteurs et textes littéraires sont des repères dans le champ descriptif des connaissances générales et servent de guide de savoir-vivre conforme à une certaine morale, à un ordre social. Ils ont explicitement une fonction didactique, puisque, offerts comme sujets d'exercice de traduction, puis d'analyse, ils sont aussi dès le début présentés comme destinés à développer chez l'élève, certaines compétences: l'élève est inscrit dans un parcours normatif; en apprenant par cœur (II: 165) certains textes 
littéraires proposés, des valeurs morales et des principes de comportement lui sont présentés comme des modèles de conduite à imiter. Le message que contient le texte littéraire à traduire s'adresse à l'élève, directement interpellé comme dans l'exemple suivant: "Souvenez-vous donc toujours de ce vers, et apprenez-le par cœur: "Un bienfait reproché tient toujours lieu d'offense" (Racine)» (II: 165). Les phrases d'auteurs littéraires (Montesquieu, Masstilon, Fénelon ...) qui sont proposées à l'analyse et qui donc illustrent des formes grammaticales sont destinées à entrer dans la mémoire de l'élève et fabriquent/constituent un répertoire de mises en condition, de jugements, de règles de comportement et de morale, comme en témoignent les verbes injonctifs (on doit, ... devrait, n'attends pas ...); ces phrases facilement mémorisables d'autant plus qu'il y est porté attention par l'analyse grammaticale, sont un catalogue d'affirmations qui relèvent plus de la croyance que du savoir et disent la norme valorisée par la société:

«Ceux de qui la conduite offre le plus à rire sont toujours sur autrui les premiers à médire. Molière» (IV: 187).

29 Le principe d'imitation des classiques -que nous avons vu s'appliquer à la for mes'applique donc aussi au comportement de l'élève, interpellé et guidé directement par Baudet et indirectement par les textes signés d'auteurs consacrés ${ }^{16} q u i$ s'adressent au lecteur; ces textes évaluatifs "Quiconque ... est...» et injonctifs «Appliquez-vous à ...» (III: 54) portent sur un sujet moral et présentent par généralisation un type de comportement loué ou condamné et avec lequel l'élève doit s'identifier. Le texte littéraire, souvent une formule impersonnelle générale qui statue sur un comportement, valorisé positivement ou négativement, porte en lui-même un message que l'élève à qui il s'adresse peut mettre en pratique; en ce sens, il est explicatif du point de vue linguistique, informatif du point de vue culturel, injonctif du point de vue individuel. La toute première citation d'un texte littéraire donnée à traduire comme texte de version est de Boileau (II: 98); elle s'adresse Aux Babillards et conseille la modération dans la forme de l'élocution et le contenu du discours:

«Tout ce qu'on dit de trop est fade et rebutant, L'esprit rassasié le rejette à l'instant».

La dernière version du volume III (III: 202), elle aussi de Boileau, complète cette injonction:

« Hâtez-vous lentement; et sans perdre courage.

Vingt fois sur le métier remettez votre ouvrage.

Polissez le sans cesse et le repolissez;

Ajoutez quelquefois, et souvent effacez. » (Boileau)

31 Voltaire est utilisé dans le même sens: «L'honneur, la probité, le sens et la raison demandent qu'on s'applique avec attention à remplir ses devoirs à ne nuire à personne» (III: 164).

32 Le guide de vie que propose Baudet indirectement est conforme à la tradition classique et antique; les dernières paroles de Socrate sont données à traduire dans un texte de Barthélémy: «Tout homme qui, renonçant aux voluptés, a pris soin d'embellir sa vie, non d'ornemens qui lui sont propres, tels que la justice, la tempérance et autres vertus, doit être plein d'une entière confiance, et attendre paisiblement l'heure de son trépas » (IV: 180). C'est la dernière version du volume IV.

33 La morale classique est ainsi portée au long des livres d'exercices, contre les vices comme l'amour-propre «dangereux ennemi» pour Madame Necker, et pour les vertus; celles de justice, tempérance et modération reviennent fréquemment dans les textes et 
sont valorisées comme menant à la sagesse, à laquelle tout homme devrait aspirer. L'adresse aux apprenants est explicite; le message didactique reprend et inclut le message littéraire: «"Vivre, ce n'est pas respirer; c'est agir: c'est faire usage de nos organes, de nos sens, de nos facultés." J.J.Rousseau. Faisons donc usage de nos facultés et surtout de nos facultés morales. Souvenons-nous que: « La mort ne surprend point le sage: il est toujours prêt à partir. » (La Fontaine, II: 170).

En apprenant la langue française, l'élève hollandais acquiert ainsi un savoir sur le bien, le beau, l'harmonieux, sur les règles d'une bonne morale sociale. Baudet, en citant les auteurs, s'inscrit dans la tradition classique qui veut que «la fin principale de la poésie est de profiter ... en purifiant les mœurs, par les instructions salutaires, qu'elle fait profession de donner à l'homme» (Rapin, Réflexions, dans Kibedi-Varga, 1990: 157).

fable, considérée comme un bon moyen de faire passer le message à transmettre, à savoir, quel est le comportement social idéal, est fortement représentée, tant dans son entier que par des citations courtes. Les fables de La Fontaine, considérées comme un livre de morale ne sont pas présentes dans leur ensemble; elles font l'objet du choix de poésies dans le recueil approprié tandis qu'ailleurs La Fontaine est cité abondamment mais à la mesure des phrases. Si l'on en croit le Père Le Bossu (1631-1680), la fonction de la fable est complexe; Baudet jouerait ainsi sur plusieurs tableaux en utilisant les exemples pris dans les fables qui permettent de ramener «à un principe commun le récit et le discours, la poétique et la rhétorique» (Kibedi-Varga, 1990: 36-37). Le linguistique et le culturel sont ici tous deux servis par la littérature. Pour Le Bossu (Traité du poème épique, Livre I: 26 sq.), «la première chose par où l'on doit commencer pour faire une fable est de choisir l'instruction et le point de morale qui lui doit servir de fond, selon le dessein et la fin que l'on se propose» (Kibedi-Varga, 1990: 140). Le point de morale pourrait être la structure profonde du texte, retenu aussi pour ses qualités formelles.

Le patrimoine littéraire français classique est vecteur de consignes morales pouvant instaurer un savoir-faire; il est aussi porteur d'un bagage culturel considéré comme un savoir nécessaire. Aux textes littéraires classiques s'ajoutent en effet bon nombre de textes des encyclopédistes, scientifiques et naturalistes du $18^{\mathrm{e}}$ siècle. Les textes de grands savants (selon le classement Larousse), comme La-place (sujet de version dès le volume II, traitant du soleil) et Cuvier, d'hommes de science reconnus comme étant des auteurs de littérature française comme Buffon, Bernardin de Saint-Pierre, Fléchier, Delille ou Raynal, sont donnés à traduire. Ils portent tantôt sur un savoir dans le domaine de l'histoire naturelle et la géographie, tantôt sur la philosophie de la vie. On pourrait dire qu'il est question de l'homme dans la nature, de son rapport avec les végétaux qui peuvent être vénéneux comme le mancenillier dont parle Raynal, les animaux apprivoisés comme l'autruche au contraire de l'hirondelle comme les compare Bernardin de Saint-Pierre, et l'environnement proche ou lointain (les Hollandais et les colonies vus par Raynal, Madras traité par Laplace). Ainsi l'élève apprendra par Raynal, Laplace ou Cuvier ce qu'est l'arbre d'où on tire le clou de girofle ou la noix de muscade, d'où vient la lumière des astres ou encore comment circulent les eaux à la surface de la terre (IV: 77-78, 168-170). Les textes de Fléchier, Buffon, Voltaire sont des considérations générales sur la vie. Outre les sciences exactes et naturelles, l'histoire a une place qui semble devenir plus grande au fil des volumes; elle tend même à remplacer la littérature aux volumes VII et Vin. Les sujets de thèmes y présentent des personnages importants par la pensée, la science, l'action politique ou militaire dans 
l'histoire. Une certaine philosophie de la vie apparaît dans la valorisation de comportements simples face à la vie qui consistent à être conscients de la relativité, de la fragilité, de la complexité de la vie humaine qui domine sur la vie animale; ainsi Fléchier oppose la noblesse d'esprit à la vanité, déplore le détournement des honneurs au profit de l'ambition et Buffon accuse les pratiques excessives qui anéantissent l'homme. Le beau linguistique et le bien moral sont tous deux cautionnés par les auteurs: «c'est la vertu que j'aime» (Boileau, IV: 179). La vérité est présentée comme la condition nécessaire à l'exercice des bonnes mœurs et la science est donnée comme garante du bon comportement des populations: «Tous les maux qu'entraîne l'ignorance se reproduiraient promptement si la lumière des sciences venait à s'éteindre» (V; 171).

On ne peut pas voir dans les Exercices la contribution à une histoire littéraire, car les auteurs cités ne le sont ni de façon systématique, ni selon un ordre chronologique quelconque. Il n'y a aucune information sur leur vie ou leur œuvre. Il n'y a pas non plus de justification des choix de Baudet dans la sélection faite parmi les auteurs classiques et encyclopédistes français, ni concernant des critères linguistiques, ni sur d'autres critères littéraire, moral, ou intellectuel. Ce qui semble importer, c'est le contenu des textes, leur portée morale. On y retrouve grosso modo les mêmes auteurs que dans les manuels d'apprentissage de la langue française qui seront utilisés dans les pays européens, en Italie en particulier, à la fin du $19^{\mathrm{e}}$ siècle; on n'y trouve en revanche ni auteur libertin, ni dramaturge osé. Parmi les encyclopédistes, le choix est-il une indication de l'intérêt et de la compétence de Baudet, limitée à certaines sciences?

A en juger par les citations de La Fontaine: «Qu'un ami véritable est une douce chose!» ou de Boileau:

Concluons qu'ici bas le seul honneur solide, C'est de prendre toujours la vérité pour guide; De regarder en tout la raison et la loi; D'être doux pour tout autre et rigoureux pour soi: d'accomplir tout le bien que le ciel nous inspire; Et d'être juste enfin: ce mot seul veut tout dire.

Baudet utilise l'exemple littéraire à des fins didactiques. La référence littéraire est là pour justifier de sa compétence; en fait, il inscrit son faire dans un cadre universellement reconnu et les écrivains classiques lui servent de caution pédagogique, renvoyant à un canon stylistique et à un canon socio-culturel.

Les auteurs classiques, surtout ceux des $17^{\mathrm{e}}$ et $18^{\mathrm{e}}$ siècles, sont pris comme modèle d'expression linguistique, tant du point de vue du style que de la correction grammaticale. L'apprentissage de la langue française pour les jeunes Hollandais de la première moitié du $19^{\mathrm{e}}$ siècle passe donc par l'imitation de la forme pure et élégante des écrivains classiques. Ceux-ci, et leur enracinement dans la philosophie humaniste et la sagesse de Montaigne, sont aussi pris comme modèles pour l'éducation morale et sociale des jeunes apprenants; les textes donnés en exemple comportent en effet des repères normatifs pour un comportement social explicitement valorisé et à imiter. 


\section{NOTES}

1. «Le corbeau et le renard» (fab 2, liv. 1), «Le loup et l'agneau» (fab 10, liv. 1) et «Le laboureur et ses enfants» (fab 9, liv. 5).

2. Racine: «Idée de Dieu», Boileau: «Dans le monde il n'est rien de beau que l'équité ...», La Fontaine: «Ni l'or, ni la grandeur ne nous rendent heureux ...».

3. Deventer J. H. de Lange, (1834-1844). Fransche cefeningen met de noodige spraakkunstige aanwijzingen en ophelderingen, ten dienste der Nederlandsche Jeugd. Les six premiers volumes sont parus de 1834 à 1836, le volume 7 en 1842 et le volume 8 en 1844 .

4. $6^{\mathrm{e}}$ édition en 1845 , Deventer J. de Lange, $8 ; 32$ pages dont 29 pages de liste de mots et 3 pages de petits textes groupés en 10 paragraphes.

5. Ce manuel, «contenant les principaux éléments d'une bonne prononciation», comprend des listes de mots et de phrases, puis une série de courts textes en français (68-74, et «La petite fille négligente», 74-79) sans aucune référence à un auteur ou à un texte littéraires; avant-propos signé par l'auteur et daté d'Utrecht 24 septembre $1845,2^{\mathrm{e}}$ éd. 1865.

6. $I^{\mathrm{e}}$ partie, 48 pages, juin 1850 , d'après l'avant-propos; $2^{e}$ partie, 96 pages; la $2^{\mathrm{e}}$ édition date de 1857.

7. Chaque volume sera désigné par le chiffre romain correspondant.

8. L'anthologie Beautés de la littérature française de Louis Paban, qui date des années 1830 en Suède, est un recueil de textes choisis qui offre les mêmes auteurs des $1 T$ et $18^{\mathrm{e}}$ siècles que les Exercices de Baudet; en revanche on y trouve des poètes du $19^{\mathrm{e}}$ siècle; voir l'étude de Hammar, E. (1994) (dans Documents S.I.H.F.L.E.S. 14, Paris, pp. 110-117.

9. Basedow, J. B. (1774). Das Elementarwerk, \114, publié la même année en français sous le titre Manuel Élémentaire dÉducation; voir l'étude de Reinfried, M. (1990). «Les origines de la méthode directe en Allemagne», dans Documents S.I.H.F.L.E.S., 6, Paris, pp. 126-156.

10. Voir (1993): Documents, n. ${ }^{\circ} 12$, pp. 5-10

11. Reinfried, M. (1990): Documents, n. ${ }^{\mathrm{a}}$ 6, p. 126.

12. C'est nous qui soulignons

13. Kibedi-Varga, Aron (1990): Les poétiques du classicisme, théorie et critique àl'âge classique, Paris, Aux Amateurs de livres.

14. C'est le cas à partir du volume IV.

15. Voir volume VIII, 8-15.

16. Les écrivains dont les textes sont utilisés pour les Exercices peuvent être qualifiés de portraitistes des mœurs, Bossuet et Fénelon dans un registre d'éducation religieuse, Racine, Corneille, La Bruyère, La Rochefoucauld pour la typologie des caractères moraux et sociaux, La Fontaine et Boileau pour la notion de bonheur individuel et d'harmonie sociale.

\section{AUTEUR}

\section{MARIE-CHRISTINE KOK ESCALLE}

Université d'Utrecht 